\title{
Efficacy and safety of adalimumab in ankylosing spondylitis
}

\section{Aziza Mounach Abdellah El Maghraoui}

Rheumatology Department, Military Hospital Mohammed V, Rabat, Morocco
Correspondence: Abdellah El Maghraoui Rheumatology Department, Military Hospital Mohammed V, PO Box 1018 , Rabat, Morocco

Tel +2I2 537716805

Fax +212537716805

Email aelmaghraoui@gmail.com
This article was published in the following Dove Press journal:

Open Access Rheumatology: Research and Reviews

13 August 2014

Number of times this article has been viewed
Abstract: Ankylosing spondylitis (AS) is the most common and most severe subtype of spondyloarthritis. It also may be an outcome of any of the other spondyloarthritis subtypes. AS preferentially affects the sacroiliac joints and the tip of the column, with a tendency to later ankylosis. Peripheral joints, enthesis, and other extra-articular involvement may be observed. Tumor necrosis factor (TNF) inhibitors are now well-established, effective drugs in the treatment of AS symptoms. Adalimumab, which is a fully human monoclonal antibody that binds to and neutralizes TNF, has demonstrated efficacy in treating AS symptoms, including axial involvement, peripheral arthritis, enthesitis, uveitis, gut involvement, and psoriasis. Furthermore, adalimumab has showed an overall acceptable safety profile. In this paper, we review the efficacy and safety profile of adalimumab in the treatment of AS, and discuss its differences from the other anti-TNF drugs reported in the literature.

Keywords: ankylosing spondylitis, spondyloarthritis, adalimumab, tumor necrosis factor- $\alpha$

\section{Introduction}

Ankylosing spondylitis (AS) is a chronic rheumatic disease which affects frequently the axial skeleton, the peripheral joints, and the entheses. AS is the major subtype of the spondyloarthritides (SpA), a group of inter-related disorders that share features, including inflammatory back pain, asymmetrical oligoarthritis of the lower limbs, enthesitis that sometimes leads to ankylosis, and specific organ involvement (uveitis, bowel disease, psoriasis, lungs interstitial fibrosis, etc). The group is strongly genetically linked to the presence of the human leukocyte antigen B27 (HLA-B27) antigen.

Around the middle of the 1990s, it was shown that tumor necrosis factor (TNF)- $\alpha$, a proinflammatory cytokine, was overexpressed in the sacroiliac joints of patients with AS. ${ }^{1}$ Knowledge of the role of TNF has provided a strong rationale for the design and use of anti-TNF drugs in AS. The TNF inhibitors have moved up the SpA treatment ladder primarily because they can control axial inflammation, along with the wide spectrum of manifestations in other domains, including the skin, peripheral joints, entheses, tendons, eye, and gut.

The primary target of treatment is remission of the disease or, at least, if this is not possible, a state of low disease activity, improving the symptoms and preventing structural damage. ${ }^{2}$ The Assessment of SpondyloArthritis International Society (ASAS) recommends use of TNF inhibitors in patients with AS, if they have active disease and have not improved with conventional treatment. ${ }^{3}$ Five anti-TNF agents are currently approved for spondyloarthritis, including four monoclonal antibodies 
(ie, infliximab, adalimumab, certolizumab pegol, and golimumab) and a soluble TNF receptor, etanercept. ${ }^{46}$

Adalimumab (Humira ${ }^{\circledR}$; Abbott Laboratories, Abbot Park, IL, USA), a fully human monoclonal antibody that binds to and neutralizes TNF, is approved for the treatment of AS, psoriatic arthritis, rheumatoid arthritis, psoriasis, juvenile idiopathic arthritis, and Crohn's disease in many countries. Large, randomized, placebo-controlled clinical trials in patients with AS have shown impressive short-term improvements in spinal pain, function, and inflammatory markers with adalimumab, when self-administered subcutaneously every other week. Long-term studies have demonstrated the sustained efficacy of adalimumab, with a safety profile comparable with that of other anti-TNF agents.

In this review, we discuss the results of the major adalimumab clinical trials, as well as observational studies in AS. However, we did not systematically review the literature, or formally assess the quality of the selected studies.

\section{Pharmacology of adalimumab}

Adalimumab is a full-length, bivalent, monoclonal immunoglobulin G1-kappa isotype (IgG1-א) antibody with a molecular weight of $150 \mathrm{kD}$, which specifically targets TNF $\alpha$, both soluble (sTNF $\alpha$ ) and membrane-bound (mTNF $\alpha$ ). It has been developed with a phage display technique and produced in a Chinese hamster ovarian cell line. It is constituted from completely human IgG1- $\kappa$ sequences and is indistinguishable from human $\operatorname{IgG1.7}$ The main mechanism of action for adalimumab is that of blocking the interaction of TNF $\alpha$ with the p55 and p75 cell surface TNF receptors. Adalimumab binds to sTNF $\alpha$ molecules (which have the potential to form multimeric complexes), which prevent sTNF $\alpha$ from binding to the natural TNF $\alpha$ receptors. Alternatively, it binds to mTNF $\alpha$ molecules, which have the potential to cross-link and reverse intracellular signaling. The efficacy of adalimumab is mediated through different mechanisms: it may neutralize sTNF $\alpha$ and mTNF $\alpha$ directly, induce apoptosis and suppress cytokines through reverse mTNF $\alpha$-mediated signaling, and induce antibody-dependent cell-mediated cytotoxicity and complement-dependent cytotoxicity, directed against cells expressing $\mathrm{mTNF} \alpha$. After a single subcutaneous injection of $40 \mathrm{mg}$, to healthy adults, the absorption of adalimumab is slow, with a maximal serum concentration of $4.7 \pm 1.6 \mu \mathrm{g} / \mathrm{mL}$, attained after $131 \pm 56$ hours. The average absolute bioavailability is $64 \% .^{8}$ The drug is considered stable for up to 24 months at $2^{\circ} \mathrm{C}-8^{\circ} \mathrm{C}$. The terminal half-life is about 2 weeks (range 10-20 days), after a $40 \mathrm{mg}$ dose, and the total body clearance is $0.18-0.27 \mathrm{~mL} /$ minute.

\section{Efficacy of adalimumab in AS Efficacy outcome measures used in the studies}

Disease activity, physical function, and mobility are usually evaluated in patients with AS using the Bath Ankylosing Spondylitis Disease Activity Index (BASDAI), ${ }^{9}$ Bath Ankylosing Spondylitis Functional Index (BASFI),${ }^{10}$ and Bath Ankylosing Spondylitis Metrology Index (BASMI), ${ }^{11}$ respectively. In 2001, the ASAS developed new outcome measures and recommended their use in trials of symptom-modifying therapy. ${ }^{12}$ The ASAS 20 improvement criteria summarize changes in four domains: physical function, pain, patient global assessment, and inflammation. A positive response is defined as $20 \%$ or greater improvement and a net improvement of one unit on a ten-unit scale, in each of three domains, with no worsening in the fourth. An ASAS 40 response is defined as $\geq 40 \%$ improvement of at least two units in each of three domains, with no worsening in the fourth. Other outcome measures include: ASAS Partial Remission (PR), defined as a score less than two units in each domain, and ASAS 5/6, defined as $20 \%$ improvement in five of six domains, adding C-reactive protein and lateral vertebral flexion. More recently, the ASAS, by analogy to rheumatoid arthritis, validated the Ankylosing Spondylitis Disease Activity Score ${ }^{13}$ (ASDAS), which includes $\mathrm{C}$-reactive protein and some questions from the BASDAI. This score showed a highly discriminatory capacity for assessing disease activity in AS.

\section{Efficacy in AS}

Adalimumab began clinical trials in 1997, and the US Food and Drug Administration (FDA) approved it for the treatment of AS in 2006. The main randomized, clinical trials analyzing the efficacy of adalimumab in AS are summarized in Table 1. The pivotal trial was the ATLAS study (Adalimumab Trial Evaluating Long-Term Safety and Efficacy for Ankylosing Spondylitis), ${ }^{14}$ a 24-week, randomized, double-blind, placebo-controlled trial of 315 AS patients with active disease despite conventional treatment. The study compared adalimumab $40 \mathrm{mg}$ every other week to placebo. The primary endpoint for efficacy assessment was the percentage of patients achieving an ASAS 20 response. The results showed that patients taking adalimumab met the primary endpoint at 12 weeks significantly more frequently than patients taking placebo ( $58 \%$ versus $21 \%$, respectively; $P=0.001)$. The onset of action was more rapid, with the benefits of adalimumab use seen by week 2 (42\% versus $16 \% ; P=0.001)$. Even patients with total spinal ankylosis achieved ASAS 20 responses at week 12 (3/6 versus 0/5), 
Table I Main randomized clinical trials analyzing the efficacy of adalimumab in ankylosing spondyloarthritis

\begin{tabular}{|c|c|c|c|c|c|c|}
\hline Clinical trial & $\begin{array}{l}\text { Disease } \\
\text { duration (years) }\end{array}$ & $\begin{array}{l}\text { Patients } \\
\text { (N) }\end{array}$ & $\begin{array}{l}\text { Mean age } \\
\text { (years) }\end{array}$ & $\begin{array}{l}\text { HLAB27 } \\
\text { (N) }\end{array}$ & $\begin{array}{l}\text { Treatment } \\
\text { duration (weeks) }\end{array}$ & Endpoints \\
\hline Van der & A 11.3 & A 208 & A 41.7 & A 163 & $12 / 24$ rescue & Primary: ASAS20 at week 12 \\
\hline Heijde et a ${ }^{22}$ & P 10.0 & $P 115$ & P 43.4 & P 85 & & $\begin{array}{l}\text { Secondary: ASAS20 at week } 24 \text { and } \\
\text { multiple measures of disease activity, } \\
\text { spinal mobility, and function, as well as } \\
\text { ASAS partial remission }\end{array}$ \\
\hline \multirow[t]{2}{*}{ Lambert et al ${ }^{17}$} & A 14.5 & A 38 & A 41.9 & NR & 12 & Primary: ASAS20 response at week 12 \\
\hline & P I2.I & P 44 & P 40.0 & & & Secondary: SPARCC scores \\
\hline \multirow[t]{2}{*}{ Haibel et a ${ }^{38}$} & A 7 & A 22 & A 38 & A 59 & 12 & Primary: ASAS40 \\
\hline & P 8 & P 24 & P 37 & P 75 & & $\begin{array}{l}\text { Secondary: ASAS20, BASDAI50, ASAS } \\
\text { partial remission, HRQOL }\end{array}$ \\
\hline \multirow[t]{2}{*}{ Hu et $\mathrm{al}^{18}$} & A 7.4 & A 26 & A 28 & & 24 & BASDAI, BASFI, CRP, ASDAS \\
\hline & P 7.6 & P 20 & P 27.4 & & & \\
\hline \multirow[t]{2}{*}{ Sieper et al ${ }^{37}$} & A 10.1 & A 91 & A 37 & A 75 & 12 & ASAS20, ASDAS, BASDAI, \\
\hline & P I0.I & P 94 & P 38.4 & P 70 & & BASDAI50 \\
\hline \multirow[t]{2}{*}{ Huang et $\mathrm{a}^{20}$} & A 8.1 & A 229 & A 30.1 & A 219 & 12 & $\begin{array}{l}\text { Primary: ASAS20 response criteria at } \\
\text { week } 12\end{array}$ \\
\hline & P 7.7 & $P \| I 5$ & P 29.6 & P 109 & & $\begin{array}{l}\text { Secondary: ASAS40, ASAS5/6, } \\
\text { high-sensitivity C-reactive protein } \\
\text { (hs-CRP), BASDAI50, disease activity, } \\
\text { pain and spinal mobility }\end{array}$ \\
\hline
\end{tabular}

Abbreviations: A, adalimumab; ASAS, Assessment in SpondyloArthritis international Society; ASDAS, Ankylosing Spondylitis Disease Activity Score; ASQol, ankylosing spondylitis quality of life; BASDAI, Bath Ankylosing Spondylitis Disease Activity Index; BASFI, Bath Ankylosing Spondylitis Functional Index; CRP, C-reactive protein; HRQOL, health-related quality of life; NR, not reported; P, placebo; RCT, randomized clinical trials; SF-36, short-form; SPARCC, Spondyloarthritis Research Consortium of Canada.

although, due to low patient numbers, the difference did not reach statistical significance. Efficacy was also demonstrated for enthesitis, using the Maastricht Ankylosing Spondylitis Enthesitis Score $^{15}$ (2.7 versus 1.3; $\left.P=0.02\right)$. The differences in outcome scores were maintained throughout the duration of the trial ( 24 weeks).

In 2009, Rudwaleit et al reported the results of a 12-week adalimumab treatment in 1,250 patients with active AS, in a multinational, open-label study. Patients achieved BASDAI 50, ASAS 40, and ASAS PR in 57.2\%, 53.7\%, and 27.7\% of cases respectively. The researchers identified four factors that were strongly associated with BASDAI 50, ASAS 40, and ASAS PR responses: younger age, elevated CRP levels, presence of HLA-B27, and anti-TNF naïvety. ${ }^{16}$

Lambert et al, ${ }^{17}$ in a randomized, double-blind, placebocontrolled trial of patients with active AS, studied the efficacy of adalimumab in reducing spinal and sacroiliac (SI) joint inflammation, using magnetic resonance imaging (MRI). Spinal and SI joint inflammation were assessed using the Spondyloarthritis Research Consortium of Canada (SPARCC) MRI index. The spine SPARCC score decreased in patients taking adalimumab, while it increased in the placebo group (mean decrease of $53.6 \%$ versus mean increase of $9.4 \%$, from baseline, respectively; $P<0.001$ ). The SI joint SPARCC score also decreased significantly from baseline, in both groups (mean $52.9 \%$, in adalimunab group, versus $12.7 \%$, in placebo group; $P=0.017$ ). The response in the adalimumab group was maintained at week 52. Another recent, randomized, controlled trial has shown obvious improvement in MRI inflammation measurements (significant decrease in lumbar spine and SI joint SPARCC scores) in active patients with AS, treated with adalimumab for 12 weeks. ${ }^{18}$ Another open-label study of adalimumab in AS used a semiquantitative approach to scoring SI joint inflammation (0-12 scoring range), but did not show a significant change in MRI score after treatment, compared with baseline, which may reflect the small sample size and/or lack of responsiveness of the scoring system. ${ }^{19}$

Recently, Huang et al evaluated adalimumab in a sample of 344 Chinese patients with AS, in a randomized, controlled trial. At week 12, adalimumab resulted in a significantly greater percentage of ASAS 20 responders than placebo $(67.2 \%$ versus $30.4 \%$, respectively; $P<0.001)$. The percentages of patients achieving ASAS 40, ASAS 5/6, and ASDAS inactive disease were significantly greater with adalimumab than placebo, at week $12(P<0.001$, for all comparisons $){ }^{20}$

\section{Quality of life}

The symptomatic and structural severity of AS is responsible for substantial functional limitations and impairment 
of quality of life. Adalimumab has demonstrated short-term improvement in clinical signs and symptoms, physical function, and health-related quality of life, in patients with active AS. ${ }^{21}$ Maintenance of improvement in quality of life outcomes has been demonstrated with adalimumab in patients who continued in open-label extension trials. ${ }^{22-24}$

\section{Efficacy in extra-articular manifestations}

Prevalence and severity of extra-articular involvement of AS vary widely across the studies. The most common manifestations are uveitis, bowel disease, lung, heart, skin, bone, and kidney involvement. ${ }^{25}$ Adalimumab has shown its effectiveness in reducing acute uveitis flares in patients with AS. ${ }^{26}$ The efficacy of TNF inhibitors in reducing acute uveitis flares was confirmed in a retrospective study of patients with $\mathrm{SpA}$, which demonstrated a clear difference between etanercept and the anti-TNF antibodies (infliximab and adalimumab). ${ }^{26}$ While the incidence of uveitis did not change with etanercept (54.6/100 patient-years [PY] versus 58.5/100 PY; $P=0.92$ ), it was significantly decreased with the anti-TNF antibodies (Infliximab: 47.4/100 PY versus 9.0/100 PY; $P=0.008$. Adalimumab: 60.5/100 PY versus $0 / 100$ PY; $P=0.04)$. An open-label study demonstrated that adalimumab may decrease the flare rates by $50 \%$ in patients with AS $(n=1,250){ }^{27}$

Nine trials were evaluated in a meta-analysis ${ }^{28}$ of trials of TNF inhibitors in AS patients (1,130 patients included in seven placebo-controlled and two open-label trials), which evaluated the incidence of flares or new onset of inflammatory bowel disease (IBD). The incidence rates for flares or new onset of IBD during treatment with adalimumab and placebo were 2.3/100 PY and 1.3/100 PY, respectively. Infliximab showed a significant superiority over etanercept $(P=0.001)$ and adalimumab $(P=0.02)$, while no significant difference between placebo and the TNF inhibitors could be shown. Furthermore, in AS patients with a history of IBD flares, the recurrence of flares was significantly greater with etanercept $(\mathrm{RR} \times 18)$ and adalimumab $(\mathrm{RR} \times 4.2)$ than with infliximab. However, it is worthy of note that data with adalimumab were quite limited, due to the lower total period of exposure (132.3 PY versus 618 PY, for infliximab, and 625.4 PY, for etanercept). An open-label study with adalimumab $(n=1,250)$ found that of the $4.7 \%$ of patients who had symptomatic IBD at baseline, $20 \%$ reported no IBD interference in the past 7 days at baseline, and $48 \%$ reported this response at 12 weeks. Moreover, it is recommended to administer adalimumab at an initial 'induction' dose of 80 $\mathrm{mg}$, when given for Crohn's disease. ${ }^{29}$ However, in ulcerative colitis, the efficacy of adalimumab is still unclear. Although the scientific basis remains uncertain, ${ }^{8}$ several hypotheses have been advanced to explain the difference in clinical efficacy profiles. It has been suggested that pharmacokinetic differences (ie, differences in the abilities to achieve adequate serum concentrations) may play a role. The differences in tissue penetration (ie, differences in ability to achieve therapeutic concentrations in inflammatory microenvironments) may be another explanation. Finally, mechanistic differences (ie, differences in ability to bind to TNF and induce therapeutic effects) have been pointed out. ${ }^{30}$

Adalimumab is registered for treating psoriasis and psoriatic arthritis, with similar reported efficacy to infliximab and etanercept. ${ }^{31-34}$ However, it is noteworthy that $1.5 \%-5 \%$ of patients treated with TNF inhibitors may present a paradoxical onset or exacerbation of psoriasis during treatment. ${ }^{35}$

\section{Efficacy on non-radiographic axial spondyloarthritis}

Recently, the ASAS group published new classification criteria for axial spondyloarthritis, including patients with and without radiographic sacroiliitis. ${ }^{36} \mathrm{~A}$ multicenter study in patients with axial SpA, called ABILITY-1, ${ }^{37}$ demonstrated the efficacy of adalimumab in patients with non-radiographic axial SpA, with ASAS 20 and ASAS 40 response rates significantly higher at week 12 , in patients taking adalimumab, compared with placebo (52\% versus $31 \%$, and $36 \%$ versus $15 \%$, respectively). Furthermore, adalimumab efficacy was also found to be significant when other composite measures were used (ie, ASAS 70, ASAS 5/6, BASDAI 50, ASDAS, and clinical remission). Significant improvements in objective measures of inflammation were observed (ie, CRP and the SPARCC MRI scores, for both SI joints and spine). In another randomized, controlled trial of 46 patients with non-radiographic axial SpA, adalimumab showed significantly better ASAS 40 responses at week 12, compared with placebo. $^{38}$

\section{Long-term use}

In a chronic disease such as AS, it is essential to know whether and how much the treatment agents maintain their safety and efficacy over time. In this regard, several studies have confirmed that TNF $\alpha$ antagonists maintain their safety and efficacy for years in patients with AS. Among 125 patients who received 5 years of adalimumab (in an open-label, 5-year follow-up of patients from the ATLAS study), results showed that $70 \%, 77 \%, 51 \%$ and $61 \%$ achieved ASAS40, BASDAI 50, ASAS partial remission and ASDAS inactive disease, 
respectively. ${ }^{39}$ A randomized, controlled trial of adalimumab in non-radiographic axial spondyloarthritis also suggested that younger age was associated with clinical outcome parameters after 1 year of treatment. ${ }^{38}$

Arends et al studied antibody formation against TNF $\alpha$ inhibitors in $60 \mathrm{AS}$ patients. ${ }^{40}$ The study found antibodies in $30 \%$ of 20 patients receiving adalimumab. It is worthy of note that the presence of antibodies was associated with loss of efficacy and early discontinuation of the anti-TNF therapy.

\section{Efficacy on radiographic progression}

TNF inhibition has no demonstrated effect on spinal bone formation in patients with AS. Most of the studies that evaluated radiographic progression used the modified Stoke Ankylosing Spondylitis Spine Score (mSASSS) method. ${ }^{41}$ The comparison between radiographic progression in patients with AS treated with adalimumab for 2 years versus patients with AS studied in a separate, historical control group (OASIS cohort), treated with conventional non-biologic therapy, did not detect any difference in mSASSS. ${ }^{42}$ Moreover, even when the analysis included only the subset of OASIS patients who satisfied the minimum baseline disease activity requirements of the adalimumab studies, no difference was observed. Similarly, many studies reported that neither etanercept ${ }^{43}$ nor infliximab ${ }^{44}$ inhibits spinal bone formation in patients with AS after 2 years of treatment. No evident hypothesis has emerged to explain why TNF inhibition therapy does not appear to stop structural progression in patients with AS. Preventing the progression of structural damage of the spine is an important goal in the treatment of AS. As pointed out in a recent editorial, ${ }^{45}$ there are two theories regarding formation of syndesmophytes in AS. One theory postulates that inflammation and osteoproliferation are related. Another theory suggests that inflammation and repair are uncoupled phenomenons. If inflammation is indeed the principal trigger of repair responses, a strong case can be made for early and/ or prolonged treatment with anti-TNF drugs. The question of whether anti-TNF agents can interfere with new bone formation in AS is difficult to answer. Previous studies have compared patients treated with anti-TNF drugs, and whose available spine radiographs had been obtained over a similar period of time. Recently, Haroon et al reported the results of an observational study addressing the effect of anti-TNF agents on radiographic progression in AS. ${ }^{46}$ This study has shown a protective effect of TNF $\alpha$ inhibitors on radiographic progression in AS. TNF $\alpha$ inhibitors are most effective when started sufficiently early in the disease course, and when treatment is sustained for a longer period of time.
Smoking and baseline levels of inflammation continue to be important risk factors, and radiographic damage at baseline remains the strongest predictor of future radiographic progression in AS. This study has used a new definition of radiographic progression. It is the first time that patients with radiographic disease progression have been defined by a rate: an increase in the mSASSS of 1 unit/year. This is not the same as the previously used definition; a minimum increase of 2 mSASSS units over 2 years. It is a questionable definition, because it assumes a linear progression in the mSASSS at the individual level, which is often not the case.

Machado $^{45}$ has stated that it is yet to be conclusively demonstrated that anti-TNF agents can have a protective, structural effect, especially if started very early in the disease process, or if given for long periods of time, with effective suppression of inflammation. The controversy continues, and these intriguing questions will continue to challenge the spondyloarthritis scientific community.

\section{Efficacy as second-line anti-TNF therapy}

Studies assessing the efficacy of adalimumab in AS patients who have failed to respond to previous TNF inhibitors are scarce. In a large retrospective study of 1,619 AS patients on TNF inhibitors, where 38 (2.34\%) switched to adalimumab after failing etanercept or infliximab, the survival curves for adalimumab, as a second biologic agent, were significantly better than the survival curves for these patients on their first anti-TNF drug $(P<0.0001)$, regardless of the reason for switching. ${ }^{47}$

Rudwaleit et $\mathrm{al}^{48}$ reviewed the efficacy of adalimumab in patients with spondyloarthritis who had previously been treated with one or two other TNF antagonists. This study showed that, after 12 weeks of adalimumab, patients with AS who had switched from infliximab, etanercept, or both experienced clinically important improvements of their disease. However, the response rates were globally lower than those for patients without prior anti-TNF therapy (ASAS 40 and BASDAI 50 response rates were $37.7 \%$ and $40.8 \%$ versus $59.3 \%$ and $63.0 \%$, respectively). These results are not surprising, and resemble what has already been observed in patients with rheumatoid arthritis treated with adalimumab as the second or third TNF inhibitor.

\section{Safety of adalimumab in AS}

A systematic review and meta-analysis was performed recently by Wang et al, to assess the efficacy and safety of adalimumab treatment in patients with AS. ${ }^{49}$ It showed that the rates of any adverse event or injection-site reaction were significantly lower in the adalimumab group versus placebo 
group (relative risk [RR]: $1.23,95 \%$ confidence interval [CI]: $1.07-1.41 ; P=0.003$ versus RR: $4.88,95 \% \mathrm{CI}$ : $1.97-12.09$; $P=0.0006$, respectively), while no difference existed between the two groups regarding the rates of serious adverse events, infection, and drug discontinuation. However, the numbers of events were too small to draw firm conclusions. Another study, including 23,458 patients from 71 clinical trials in rheumatoid arthritis, juvenile idiopathic arthritis, AS, psoriatic arthritis, psoriasis and Crohn's disease was performed recently by Burmester et al, ${ }^{50}$ with the objective to analyze the long-term safety profile of adalimumab. Across all six therapeutic indications, serious infectious events (SIEs) were the most frequently reported serious adverse event. However, in patients with AS, no SIE rate exceeded 0.2/100 PY. No active tuberculosis or serious opportunistic infections were reported (Table 2). Since latent tuberculosis infection screening and prophylaxis were implemented in 1998 and 1999, respectively, the rate has decreased from 1.5/10 PY to 0.2/100 PY. The incidence rates of serious new onset or worsening of psoriasis was very low, at $<0.1$ events/100 PY. Regarding the other classical adverse events with TNF inhibitors, the incidence rates of demyelinating disorders, lupus-like syndrome, and congestive heart failure, across all indications, were $\leq 0.1$ events/100 PY. Rates of malignancies, across all indications, were 0.7 events/100 PY (excluding lymphoma and nonmelanoma skin cancer), 0.1/100 PY, for lymphoma, and 0.2/100 PY, for nonmelanoma skin cancer. Moreover, the observed number of deaths in patients treated with adalimumab, in rheumatoid arthritis, AS, and psoriasis clinical trials was less than expected in an age- and sex-matched

Table 2 Incidence rates of serious adverse events of interest in patients with ankylosing spondylitis $(n=1684)$

\begin{tabular}{ll}
\hline Exposure, patient-years & 1985.6 \\
Serious infections & 1.4 \\
Active tuberculosis & 0 \\
Opportunistic infections & 0 \\
Demyelinating disorder & $<0.1$ \\
Lupus-like syndrome & 0.1 \\
Congestive heart failure & 0.1 \\
New onset/worsening of psoriasis & $<0.1$ \\
Malignancies excluding lymphoma and NMSC & 0.2 \\
Lymphoma & $<0.1$ \\
NMSC & 0.3 \\
Melanoma & $<0.1$ \\
Any adverse event leading to death & $<0.1$
\end{tabular}

Notes: Rates in events/100 patient-years. NMSC includes only serious events. Adapted from Burmester GR, Panaccione R, Gordon KB, Mcllraith MJ, Lacerda AP. Adalimumab: long-term safety in 23458 patients from global clinical trials in rheumatoid arthritis, juvenile idiopathic arthritis, ankylosing spondylitis, psoriatic arthritis, psoriasis and Crohn's disease. Ann Rheum Dis. 2013;72(4):5 I7-524. ${ }^{50}$ Abbreviations: NMSC, non-melanoma skin cancer; PY, patient-years. population, while it was similar in patients with psoriatic arthritis and Crohn's disease. ${ }^{49}$

The main limitation of this review is that we did not systematically check the medical literature with prespecified inclusion criteria and quality control of the selected studies. However, the objective was to review the efficacy and safety of adalimumab in AS in a global way, whatever the design of the studies. Another limitation is that data extracted from randomized, controlled trials correspond to patients included in the adalimumab clinical trial programme, with specific inclusion and exclusion criteria. Thus, the study population was likely healthier than the wider, general patient population, with fewer comorbidities, which might have resulted in better efficacy results. Moreover, they were closely monitored at regular scheduled visits, which might have resulted in detection bias for adverse events.

\section{Conclusion}

Data from randomized clinical trials and open-label extension studies show that adalimumab is an effective treatment in patients with AS. A benefit of improved clinical outcomes is reported in many manifestations of the disease, including axial involvement, peripheral arthritis, enthesitis, uveitis, gut involvement, and psoriasis. Moreover, the efficacy of adalimumab in patients who have failed to respond to previous biologics has been evaluated in a few studies, and reported good clinical response rates. Similarly to the other anti-TNF agents, no effect on structural progression has been demonstrated, and long-term studies have showed an acceptable safety profile.

\section{Disclosure}

The authors report no conflicts of interest in this work.

\section{References}

1. Braun J, Bollow M, Neure L, et al. Use of immunohistologic and in situ hybridization techniques in the examination of sacroiliac joint biopsy specimens from patients with ankylosing spondylitis. Arthritis Rheum. 1995;38(4):499-505.

2. Smolen JS, Braun J, Dougados M, et al. Treating spondyloarthritis, including ankylosing spondylitis and psoriatic arthritis, to target: recommendations of an international task force. Ann Rheum Dis. 2014;73(1): 6-16.

3. van der Heijde D, Sieper J, Maksymowych WP, et al. 2010 Update of the international ASAS recommendations for the use of anti-TNF agents in patients with axial spondyloarthritis. Ann Rheum Dis. 2011;70(6): 905-908.

4. Braun J, van den Berg R, Baraliakos X, et al. 2010 update of the ASAS/ EULAR recommendations for the management of ankylosing spondylitis. Ann Rheum Dis. 2011;70(6):896-904.

5. Braun J, Baraliakos X, Hermann KG, et al. The effect of two golimumab doses on radiographic progression in ankylosing spondylitis: results through 4 years of the GO-RAISE trial. Ann Rheum Dis. Jun 2014;73(6): $1107-1113$. 
6. Landewe R, Braun J, Deodhar A, et al. Efficacy of certolizumab pegol on signs and symptoms of axial spondyloarthritis including ankylosing spondylitis: 24-week results of a double-blind randomised placebocontrolled Phase 3 study. Ann Rheum Dis. 2014;73(1):39-47.

7. Voulgari PV, Drosos AA. Adalimumab in the treatment of rheumatoid arthritis. Expert Opin Biol Ther. Epub 2014 March 4.

8. Tracey D, Klareskog L, Sasso EH, Salfeld JG, Tak PP. Tumor necrosis factor antagonist mechanisms of action: a comprehensive review. Pharmacol Ther. 2008;117(2):244-279.

9. Garrett S, Jenkinson T, Kennedy LG, Whitelock H, Gaisford P, Calin A. A new approach to defining disease status in ankylosing spondylitis: the Bath Ankylosing Spondylitis Disease Activity Index. J Rheumatol. 1994;21(12):2286-2291.

10. Calin A, Garrett $\mathrm{S}$, Whitelock $\mathrm{H}$, et al. A new approach to defining functional ability in ankylosing spondylitis: the development of the Bath Ankylosing Spondylitis Functional Index. J Rheumatol. 1994;21(12): 2281-2285.

11. Jenkinson TR, Mallorie PA, Whitelock HC, Kennedy LG, Garrett SL, Calin A. Defining spinal mobility in ankylosing spondylitis (AS). The Bath AS Metrology Index. J Rheumat. 1994;21(9):1694-1698.

12. Anderson JJ, Baron G, van der Heijde D, Felson DT, Dougados M. Ankylosing spondylitis assessment group preliminary definition of short-term improvement in ankylosing spondylitis. Arthritis Rheum. 2001;44(8):1876-1886.

13. Lukas C, Landewe R, Sieper J, et al. Development of an ASAS-endorsed disease activity score (ASDAS) in patients with ankylosing spondylitis. Ann Rheum Dis. 2009;68(1):18-24.

14. Revicki DA, Luo MP, Wordsworth P, et al. Adalimumab reduces pain, fatigue, and stiffness in patients with ankylosing spondylitis: results from the adalimumab trial evaluating long-term safety and efficacy for ankylosing spondylitis (ATLAS). J Rheumat. 2008;35(7):1346-1353.

15. Heuft-Dorenbosch L, Spoorenberg A, van Tubergen A, et al. Assessment of enthesitis in ankylosing spondylitis. Ann Rheum Dis. 2003;62(2): 127-132.

16. Rudwaleit M, Claudepierre P, Wordsworth P, et al. Effectiveness, safety, and predictors of good clinical response in 1250 patients treated with adalimumab for active ankylosing spondylitis. J Rheumatol. 2009;36(4): 801-808.

17. Lambert RG, Salonen D, Rahman P, et al. Adalimumab significantly reduces both spinal and sacroiliac joint inflammation in patients with ankylosing spondylitis: a multicenter, randomized, double-blind, placebo-controlled study. Arthritis Rheum. 2007;56(12):4005-4014.

18. Hu Z, Xu M, Li Q, et al. Adalimumab significantly reduces inflammation and serum DKK-1 level but increases fatty deposition in lumbar spine in active ankylosing spondylitis. Int J Rheum Dis. 2012;15(4):358-365.

19. Haibel H, Rudwaleit M, Brandt HC, et al. Adalimumab reduces spinal symptoms in active ankylosing spondylitis: clinical and magnetic resonance imaging results of a fifty-two-week open-label trial. Arthritis Rheum. 2006;54(2):678-681.

20. Huang F, Gu J, Zhu P, et al. Efficacy and safety of adalimumab in Chinese adults with active ankylosing spondylitis: results of a randomised, controlled trial. Ann Rheum Dis. 2014;73(3):587-594.

21. Davis JC Jr, Revicki D, van der Heijde DM, et al. Health-related quality of life outcomes in patients with active ankylosing spondylitis treated with adalimumab: results from a randomized controlled study. Arthritis Rheum. 2007;57(6):1050-1057.

22. van der Heijde D, Schiff MH, Sieper J, et al. Adalimumab effectiveness for the treatment of ankylosing spondylitis is maintained for up to 2 years: long-term results from the ATLAS trial. Ann Rheum Dis. 2009;68(6):922-929.

23. Botteman MF, Hay JW, Luo MP, Curry AS, Wong RL, van Hout BA. Cost effectiveness of adalimumab for the treatment of ankylosing spondylitis in the United Kingdom. Rheumatology (Oxford). 2007;46(8): $1320-1328$.

24. Wailoo A, Bansback N, Chilcott J. Infliximab, etanercept and adalimumab for the treatment of ankylosing spondylitis: cost-effectiveness evidence and NICE guidance. Rheumatology (Oxford). 2008;47(2):119-120.
25. El Maghraoui A. Extra-articular manifestations of ankylosing spondylitis: prevalence, characteristics and therapeutic implications. Eur J Intern Med. 2011;22(6):554-560.

26. Guignard S, Gossec L, Salliot C, et al. Efficacy of tumour necrosis factor blockers in reducing uveitis flares in patients with spondylarthropathy: a retrospective study. Ann Rheum Dis. 2006;65(12):1631-1634.

27. Rudwaleit M, Rodevand E, Holck P, et al. Adalimumab effectively reduces the rate of anterior uveitis flares in patients with active ankylosing spondylitis: results of a prospective open-label study. Ann Rheum Dis. 2009;68(5):696-701.

28. Braun J, Baraliakos X, Listing J, et al. Differences in the incidence of flares or new onset of inflammatory bowel diseases in patients with ankylosing spondylitis exposed to therapy with anti-tumor necrosis factor alpha agents. Arthritis Rheum. 2007;57(4):639-647.

29. Rudwaleit M, Baeten D. Ankylosing spondylitis and bowel disease. Best Pract Res Clin Rheumatol. 2006;20(3):451-471.

30. Kaymakcalan Z, Sakorafas P, Bose S, et al. Comparisons of affinities, avidities, and complement activation of adalimumab, infliximab, and etanercept in binding to soluble and membrane tumor necrosis factor. Clin Immunol. May 2009;131(2):308-316.

31. Hoy SM, Scott LJ. Etanercept: a review of its use in the management of ankylosing spondylitis and psoriatic arthritis. Drugs. 2007;67(17): 2609-2633.

32. Barra L, Pope JE, Payne M. Real-world anti-tumor necrosis factor treatment in rheumatoid arthritis, psoriatic arthritis, and ankylosing spondylitis: cost-effectiveness based on number needed to treat to improve health assessment questionnaire. J Rheumatol. 2009;36(7):1421-1428.

33. Brodszky V, Pentek M, Gulacsi L. Efficacy of adalimumab, etanercept, and infliximab in psoriatic arthritis based on ACR50 response after 24 weeks of treatment. Scand J Rheumatol. 2008;37(5):399-400.

34. Gladman DD. Adalimumab, etanercept and infliximab are equally effective treatments for patients with psoriatic arthritis. Nat Clin Pract Rheumatol. 2008;4(10):510-511.

35. Wendling D, Balblanc JC, Briancon D, et al. Onset or exacerbation of cutaneous psoriasis during TNFalpha antagonist therapy. Joint Bone Spine. 2008;75(3):315-318.

36. Rudwaleit M, van der Heijde D, Landewe R, et al. The development of Assessment of SpondyloArthritis international Society classification criteria for axial spondyloarthritis (part II): validation and final selection. Ann Rheum Dis. 2009;68(6):777-783.

37. Sieper J, van der Heijde D, Dougados M, et al. Efficacy and safety of adalimumab in patients with non-radiographic axial spondyloarthritis: results of a randomised placebo-controlled trial (ABILITY-1). Ann Rheum Dis. 2013;72(6):815-822.

38. Haibel $\mathrm{H}$, Rudwaleit M, Listing J, et al. Efficacy of adalimumab in the treatment of axial spondylarthritis without radiographically defined sacroiliitis: results of a twelve-week randomized, double-blind, placebocontrolled trial followed by an open-label extension up to week fifty-two. Arthritis Rheum. 2008;58(7):1981-1991.

39. Sieper J, van der Heijde D, Dougados M, Brown LS, Lavie F, Pangan AL. Early response to adalimumab predicts long-term remission through 5 years of treatment in patients with ankylosing spondylitis. Ann Rheum Dis. 2012;71(5):700-706.

40. Arends S, Lebbink HR, Spoorenberg A, et al. The formation of autoantibodies and antibodies to TNF-alpha blocking agents in relation to clinical response in patients with ankylosing spondylitis. Clin Exp Rheumatol. 2010;28(5):661-668.

41. Creemers MC, Franssen MJ, van't Hof MA, Gribnau FW, van de Putte LB, van Riel PL. Assessment of outcome in ankylosing spondylitis: an extended radiographic scoring system. Ann Rheum Dis. 2005;64(1):127-129.

42. van der Heijde D, Salonen D, Weissman BN, et al. Assessment of radiographic progression in the spines of patients with ankylosing spondylitis treated with adalimumab for up to 2 years. Arthritis Res Ther. 2009;11(4):R127.

43. van der Heijde D, Landewe R, Einstein S, et al. Radiographic progression of ankylosing spondylitis after up to two years of treatment with etanercept. Arthritis Rheum. 2008;58(5):1324-1331. 
44. van der Heijde D, Baraf HS, Ramos-Remus C, et al. Evaluation of the efficacy of etoricoxib in ankylosing spondylitis: results of a fifty-two-week, randomized, controlled study. Arthritis Rheum. 2005;52(4):1205-1215.

45. Machado P. Anti-tumor necrosis factor and new bone formation in ankylosing spondylitis: the controversy continues. Arthritis Rheum. 2013;65(10):2537-2540.

46. Haroon N, Inman RD, Learch TJ, et al. The impact of tumor necrosis factor alpha inhibitors on radiographic progression in ankylosing spondylitis. Arthritis Rheum. 2013;65(10):2645-2654.

47. Spadaro A, Punzi L, Marchesoni A, et al. Switching from infliximab or etanercept to adalimumab in resistant or intolerant patients with spondyloarthritis: a 4-year study. Rheumatology (Oxford). 2010;49(6): 1107-1111.
48. Rudwaleit M, Van den Bosch F, Kron M, Kary S, Kupper H. Effectiveness and safety of adalimumab in patients with ankylosing spondylitis or psoriatic arthritis and history of anti-tumor necrosis factor therapy. Arthritis Res Ther. 2010;12(3):R117.

49. Wang H, Zuo D, Sun M, Hua Y, Cai Z. Randomized, placebo controlled and double-blind trials of efficacy and safety of adalimumab for treating ankylosing spondylitis: a meta-analysis. Int J Rheum Dis. 2014;17(2): $142-148$.

50. Burmester GR, Panaccione R, Gordon KB, McIlraith MJ, Lacerda AP. Adalimumab: long-term safety in 23458 patients from global clinical trials in rheumatoid arthritis, juvenile idiopathic arthritis, ankylosing spondylitis, psoriatic arthritis, psoriasis and Crohn's disease. Ann Rheum Dis. 2013;72(4):517-524.

\section{Publish your work in this journal}

Open Access Rheumatology Research and Reviews is an international, peer-reviewed, open access journal, publishing all aspects of clinical and experimental rheumatology in the clinic and laboratory including the following topics: Pathology, pathophysiology of rheumatological diseases; Investigation, treatment and management of rheumatological diseases; Clinical trials and novel pharmacological approaches for the treatment of rheumatological disorders. The manuscript management system is completely online and includes a very quick and fair peerreview system, which is all easy to use. Visit http://www.dovepress.com/ testimonials.php to read real quotes from published authors. 